\title{
UMA NOVA EXPRESSÃO PARA A VARIÂNCIA DA VELOCIDADE TURBULENTA CONVECTIVA DERIVADA DE UM MODELO LES
}

\author{
Gervásio Annes Degrazia ${ }^{1}$, Lilian P. Moor ${ }^{1}$, Silvana Maldaner ${ }^{1}$, Silvia Beatriz Alves \\ Rolim $^{2}$, Umberto Rizza ${ }^{3}$
}

${ }^{1}$ Universidade Federal de Santa Maria, Santa Maria, RS, Brasil.

${ }^{2}$ Programa de Pós-Graduação em Sensoriamento Remoto, Universidade Federal do Rio Grande do Sul, Porto Alegre, RS, Brasil.

${ }^{3}$ Institute of Atmospheric Sciences and Climate (ISAC) of the Italian National Research Council (CNR), Unit of Lecce, Italy

\section{SUMMARY}

The aim of this study is to derive a new expression for the vertical turbulent velocity variance using LES data in a convective boundary layer.

\section{INTRODUÇÃO}

A determinação das variâncias de velocidade turbulentas na camada limite planetária (CLP) é de importância fundamental no emprego de modelos de dispersão de contaminantes. Particularmente, esta quantidade é utilizada em modelos Eulerianos e Lagrangeanos de dispersão (Rodean, 1996). Uma expressão que representa o espectro de velocidade turbulenta na camada limite convectiva (CLC) pode ser escrita como (Degrazia, 2000)

$$
\frac{n S_{i}^{E}(n)}{w_{*}^{2}}=\frac{1.06 c_{i} f \psi^{2 / 3}\left(z / z_{i}\right)^{2 / 3}}{\left(f_{m}\right)_{i}^{5 / 3}\left\{1+1.5\left[\frac{f}{\left(f_{m}\right)_{i}}\right]\right\}^{5 / 3}}
$$

onde $f=n z / U(z)$ é a frequência adimensional, $w_{*}$ é a escala de velocidade convectiva, $z$ é a altura acima do solo, $\left(f_{m}\right)_{i}$ é a frequência normalizada do máximo espectral, $z_{i}$ é a altura do topo da CLC, $\psi=\varepsilon z_{i} / w_{*}^{3}$ é a função taxa de dissipação adimensional, $c_{i}=\alpha_{i} \alpha_{u}(2 \pi k)^{2 / 3}$, $\alpha_{u}=0.50 \pm 0.05$ e $\alpha_{i}=1,4 / 3,4 / 3$ para as componentes u, v e w (Sorbjan, 1989). A integração analítica da Eq. (1) sobre todo domínio de frequência, resulta na variância da velocidade do vento, dada por: 


$$
\sigma_{i}^{2}=\frac{1,1 c_{i} \Psi_{\varepsilon}^{2 / 3} w_{*}^{2}\left(z / z_{i}\right)^{2 / 3}}{\left[\left(f_{m}\right)_{i}\right]^{2 / 3}}
$$

a Eq. (2) é descrita em termos da frequência fundamental $\left(f_{m}\right)_{i}$. Tal frequência fornece as escalas de tempo e de comprimento associadas aos turbilhões contendo a energia principal do escoamento turbulento. Geralmente, expressões para $\left(f_{m}\right)_{i}$ são quase todas obtidas a partir de relações que aproximam os dados experimentais (curvas de ajuste). Entretanto, dados experimentais deste parâmetro fundamental, conectado aos espectros de energia turbulenta, são difíceis de obter. Por esta razão, os modelos de simulações dos grandes turbilhões constituem uma ferramenta útil para se determinar o parâmetro $\left(f_{m}\right)_{i}$.

O objetivo do presente estudo é derivar uma nova formulação para a variância da velocidade vertical turbulenta na CLC. Esta expressão é formulada da Eq. (2) utilizando uma relação para $\left(f_{m}\right)_{w}$ obtida de uma simulação dos grandes turbilhões (LES). Esta nova fórmula, derivada para $\sigma_{w}^{2}$, é comparada com dados experimentais.

\section{DERIVAÇÃO DE UMA EXPRESSÃO PARA $\sigma_{w}^{2}$ A PARTIR DOS DADOS DE $\left(f_{m}\right)_{w}$ GERADOS EM UMA SIMULAÇÃO LES}

Uma expressão para o comprimento de onda do máximo do espectro de energia da velocidade vertical em uma CLC tem sido proposta por Marques Filho (2004). Esta equação, derivada de uma simulação dos grandes turbilhões, pode ser escrita como

$$
\left(\lambda_{m}\right)_{w}=1,3 z_{i}\left[1-\exp \left(-4,8 z / z_{i}\right)-0,005 \exp \left(4,8 z / z_{i}\right)\right]
$$

definindo-se $\left(f_{m}\right)_{w}=z /\left(\lambda_{m}\right)_{w}$ na equação (2) e utilizando-se $\Psi_{\varepsilon}=0,65$ e $c_{w}=0,4$, resulta uma expressão para $\sigma_{w}^{2}$ :

$$
\sigma_{w}^{2}=0,37 w_{*}^{2}\left[1-\exp \left(-4,8 z / z_{i}\right)-0.005 \exp \left(4,8 z / z_{i}\right)\right]^{2 / 3}
$$

A Figura 1 mostra o perfil vertical da $\sigma_{w}^{2}$ fornecido pela equação (4). Analisando-se este perfil, a partir de uma comparação com os valores observacionais de $\sigma_{w}^{2}$, pode-se ver que esta nova formulação reproduz razoavelmente bem os resultados medidos em toda a 
extensão vertical da CLC. Diferentemente, a expressão sugerida por Lenschow (1980) subestima os pontos experimentais na metade superior da CLC.

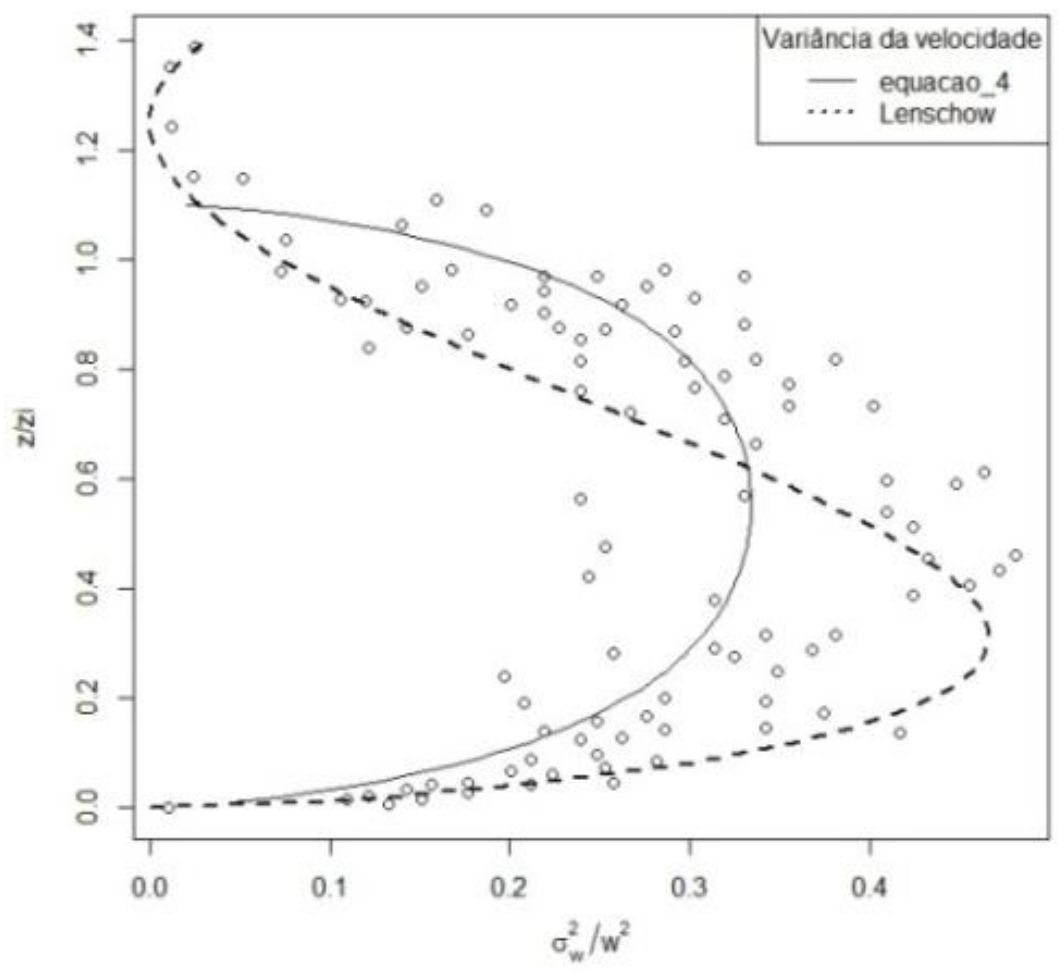

Figura 1 - Perfil vertical da variância da velocidade vertical. A curva em linha contínua é calculada a partir da expressão (4). A curva em linha pontilhada é referente a expressão proposta por Lenschow (1980). Os pontos são dados experimentais para uma CLC retirados de Caughey (1979).

\section{CONCLUSÕES}

A derivação empregou a frequência do máximo espectral obtida a partir de uma simulação dos grandes turbilhões (LES). A Eq. (4) apresenta um perfil vertical bem comportado e reproduz razoavelmente bem os pontos experimentais para as variâncias da velocidade. Pode-se concluir que a Eq. 4 pode ser empregada em modelos de dispersão para simular o campo de concentração de fontes pontuais contínuas na CLC.

\section{AGRADECIMENTOS}

Agradecimento à CAPES, CNPq e FAPERGS. 


\section{REFERÊNCIAS}

CAUGHEY, S. J., PALMER, S. G. Some aspects of turbulence structure through the depth of the convective boundary layer. Quart. J. Roy. Meteor, Soc., v. 105, p. 811-827, 1979.

DEGRAZIA, G. A., ANFOSSI, D., CARVAlHO, J., MANGIA, C., TIRABASSI, T., CAMPOS VELHO, H. Turbulence parametrization for PBL dispersion models in all stability conditions. Atmos. Environment., v.34, p.3575-3583. 2000.

LENSCHOW, D. H.; WYNGAARD, J. C.; PENNEL, W. T. Mean-field and secondmoment budgets in baroclinic, convective boundary layer. J. Atmos. Sci., v.37, p. 1313-1326. 1980.

MARQUES FILHO, E. P. Investigação da camada limite planetária convectiva com modelo LES aplicado à dispersão de poluentes. Tese de Doutorado. Instituto de Astronomia, Geofísica e Ciências Atmosféricas da USP. São Paulo. 2004.

RODEAN, H.C. Stochastic Lagrangian Models of Turbulence Diffusion. Boston: American Meteorological Society, 84p., 1996.

SORBJAN, Z., Structure of The Atmospheric Boundary Layer, Prentice Hall, New Jersey, 317p., 1989. 